\title{
Reconstruction of Limb Leads Recorded on The Subject's Torso
}

\author{
Judyta Salamon ${ }^{1}$, Zuzanna Puzio ${ }^{1}$, Łukasz Kołtowski, MD, $\mathrm{PhD}^{2}$, Grzegorz Wróblewski, $\mathrm{PhD}^{3}$, \\ Adrian Maciejewski, $\mathrm{MD}^{4}$ \\ ${ }^{1}$ Physics of Complex Systems Division, Faculty of Physics, Warsaw University of Technology, \\ 2 1st Department of Cardiology, Medical University of Warsaw, \\ ${ }^{3}$ Institute of Metrology and Biomedical Engineering, Warsaw University of Technology, \\ ${ }^{4}$ Poznan University of Medical Sciences, Poznan, Poland
}

\begin{abstract}
Standard limb electrode position may be challenging when a patient is subjected to a stress test, due to limb movement. Mason-Likar position compensates for such difficulties, as all limbs electrodes are placed on the torso. On the other hand, such recordings show a number of differences when compared to standard placement. We wanted to find a compromise between them, by placing limb leads closer together on the human body to avoid motion artifacts and using signal reconstruction to make the trace diagnostic.

Reconstruction has been performed based on conversion matrices, that were created using linear and lasso regression, polynomials fitting and Support Vector Regression (SVR).

For each method, the reconstructed signal has been compared to the one recorded in reference positions. The results of each method showed results with a mean difference between all points from $0.019 \mathrm{mV}$ to $0.045 \mathrm{mV}$. For linear regression the mean difference between $R$ wave amplitudes was $0.1 \mathrm{mV}, 0.03 \mathrm{mV}$ for $Q$ waves and $0.08 \mathrm{mV}$ for $S$ wave. The best results were achieved with the use of linear regression.
\end{abstract}

\section{Introduction}

Electrocardiogram recording is one of the most common screening test. 12-lead ECG has been widely used since 65 years, when the American Heart Association published recommendations to standardize ECG's [1]. Limb lead placement was defined even earlier by Einthoven to be placed on the wrist and ankles. In 1966, Mason and Likar proposed a modified limb leads placement, which enabled recording signals free of motion artifacts during exercise testing or when the limbs were inaccessible [2]. On the other hand, such recordings showed a number of differences to standard positioning, for example amplitude and waveform changes, causing, among others, a rightward shift of the QRS axis [3].

It has been noticed that in $36 \%$ of healthy subjects with normal ECG important abnormalities occurred [3]. Also in the same study, in patients with abnormal standard ECGs, moving the limb leads to the torso made possible MIs appear and inferior infarcts disappear. Likewise, other developed T wave or QRS axis clinically important changes.

Based on the aforementioned data, it is not possible to compare standard 12-lead ECG traces to transpositioned lead placement. To make the signals diagnostic, signal reconstruction must be performed. Most common method for signal modification is using a conversion matrix $[4,5]$. The most widely used method for conversion matrix generation is linear regression [4-6]. Other common methods include polynomial fitting [7], lasso regression [8,9] and SVR [10].

The main purpose of this study was to test the selected methods for reconstruction of the 12-lead ECG signals recorded in modified limb lead position to be comparable to the standard placement.

\section{Methods}

Signal recording were performed using two devices simultaneously (M-Trace PC, M4Medical). In both studies precordial leads were placed in standard position. Limb leads altered were transpositioned from standard 12-lead placement to a placement proposed by SmartMedics $\mathrm{Sp} \mathrm{z}$ o.o.. For male participants, upper limb leads were placed $2 \mathrm{~cm}$ above V5 and symmetrically on the right side; the lower limb leads were placed $2 \mathrm{~cm}$ below V5 and symmetrically on the right side (Figure 1a). For female participants, the limb leads were placed above and below the mid-line from V5 to V6 - Figure 1b). Differences in placement in men and women were due to differences in the anatomical structure. 


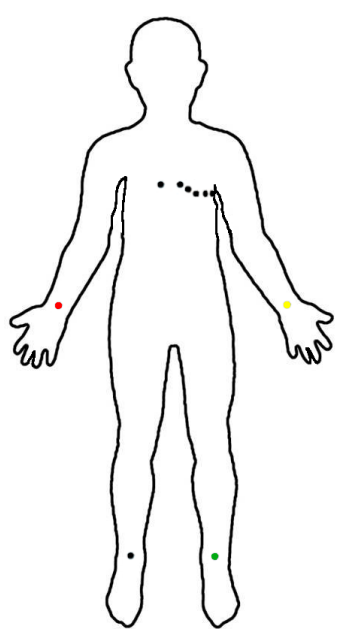

(a) Standard positions

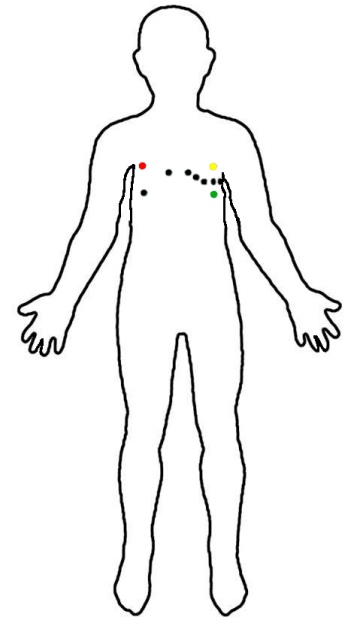

(b) Modified positions
Figure 1: Standard versus modified positions.

88 men $(25 \pm 5$ years old $)$ and 51 women $(27 \pm 8$ years old) were examined after signing informed consent. All subjects were healthy, without any cardiological history. 60 -seconds recordings were performed, based on them, averaged beats have been created.

\section{Matrix generation and testing}

Reconstruction has been performed with the use of conversion matrices, that were created using linear and lasso regression, polynomials fitting and Support Vector Regression (SVR). Generated matrices were $12 \times 12$ (reconstruction of all leads), 8x8 (without leads III, aVR, aVL, aVF). For polynomials fitting 2 nd and 3rd degree, the matrices were respectively: 12 x 91 and 12 x 455 .

Each sex provided a separate database. Recordings in each group were shuffled and divided for learning and testing sets. A k-fold Cross-Validation was performed with 10 subjects in testing set, the learning set contained the remainder. The procedure was repeated until each entry was tested (i.e. 9 folds for men, 5 folds for women). For each testing fold, the following statistical metrics were calculated:

- Difference - mean absolute difference between reference and reconstructed signals $[\mathrm{mV}]$;

- Std - square root of the average of the squared deviations;

- $R^{2}$ - coefficient of determination regression score function, best possible score is 1.0 ;

- Corr - Spearman rank-order correlation coefficient, showing how strong pair of variables are related. Correlation coefficients varies between -1 and +1 with 0 implying no correlation.

Also, the averaged differences between $\mathrm{Q}, \mathrm{R}$ and $\mathrm{S}$ waves amplitudes have been verified.

\section{Results}

For each method of matrix creation, the reconstructed signal have been compared to the one recorded in reference positions. Polynomials fitting results were unsatisfactory and haven't been further shown. Figures 2 and 3 show two examples of signal reconstruction, with standard 12-lead ECG (Reference, blue line) and signal reconstructed using linear regression and SVR respectively (Reconstructed, orange line) for two different subjects. In the two examples shown below, a very good agreement between recordings can be observed.

Statistical metrics described above are given in Table 1 for men and women groups. Averaged values of all folds for each metric have been computed. We can see the average differences between signals varies from 0.02 to 0.05 $\mathrm{mV}$. Mean $R^{2}$ value is around 0.76. Correlation values range between 0.6 for III lead, up to 0.98 for V4 lead.

Figure 4 shows averaged differences between most important waves in the ECG waveforms, that is $\mathrm{Q}, \mathrm{R}$ and $\mathrm{S}$ waves. We can see that averaged differences oscillate around $0.2 \mathrm{mV}$. No difference exceeds $0.5 \mathrm{mV}$.

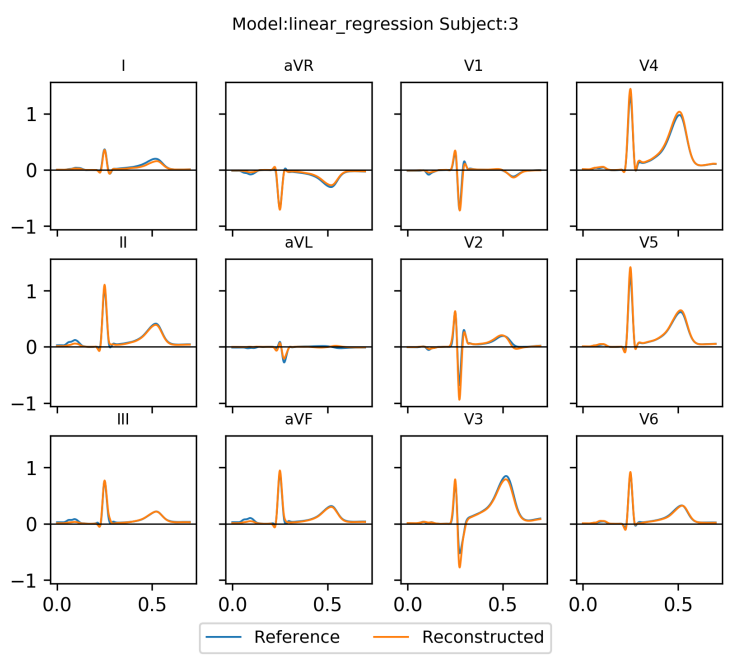

Figure 2: An example of signal reconstruction, signals obtained with linear regression. Amplitude values are in $\mathrm{mV}$.

\section{Discussion and conclusions}

In our study we tested five methods to obtain a reconstructed signal, based on signals recorded in modified limb leads position, that were placed on the torso. Signal reconstruction is necessary when electrodes are in modified positions, as amplitudes and waves morphologies have been changed. The above three methods of matrix generation 


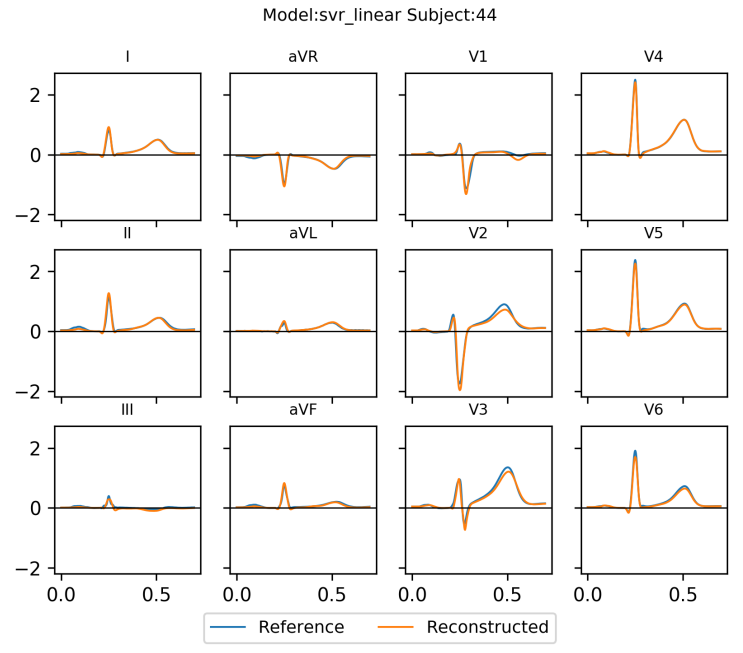

Figure 3: An example of signal reconstruction, signals obtained with SVR. Amplitude values are in $\mathrm{mV}$.

give similar outcome, but based on all statistical metrics, linear regression provided the best results.

The reconstruction worked better for precordial leads, possibly due to the fact that changing the placement of limb leads influences them less, since only the reference point for precordial leads measurement is changing (Wilson Central Terminal). In a study by Farrell et. al. changes in WCT have been estimated to be around $100 \mu \mathrm{V}$ in ML positions [11]. In our placement the changes are more prevalent, as limb leads are further from the reference positions, so the reconstruction in needed.

We can observe significantly worse results for leads III and aVL, which may be caused by low amplitudes in those leads, that compound the error. These are also the leads in which waves morphologies are highly different in the population, which may lead to difficulties in an appropriate reconstruction of those leads.

There are no visible differences between male and female groups. We can assume that proposed methods works just as well for both placements, which are forced to be introduced by anatomical differences in men and women. Further research could be performed to verify if it is possible to generate one universal matrix that would achieve satisfactory results for both populations.

Average differences between reference and reconstructed signals are small (average of $0.03 \mathrm{mV}$ ), but some differences between amplitudes of $\mathrm{Q}, \mathrm{R}$ and $\mathrm{S}$ waves could be noticed. Each of the reconstructed signals should be further compared with the reference recordings by a cardiologist and evaluated if the signals don't differ diagnostically. High correlation coefficients (around 0.88 with $\mathrm{p}$ value always below 0.01 ) indicates that the methods work well, but require further development. Also P and T waves, as well as ST segment amplitude should be compared.

\begin{tabular}{|c|c|c|c|c|c|c|c|c|c|c|c|c|c|c|}
\hline Sex & Stats & I & II & III & aVR & $\mathrm{aVL}$ & $\mathrm{aVF}$ & V1 & V2 & V3 & V4 & V5 & V6 & Mean \\
\hline \multirow{15}{*}{ 岳 } & \multicolumn{14}{|c|}{ Linear regression } \\
\hline & Diff & 0.02 & 0.04 & 0.04 & 0.03 & 0.03 & 0.04 & 0.04 & 0.04 & 0.04 & 0.02 & 0.02 & 0.02 & 0.03 \\
\hline & Std & 0.04 & 0.07 & 0.07 & 0.04 & 0.05 & 0.06 & 0.07 & 0.08 & 0.09 & 0.04 & 0.04 & 0.04 & 0.06 \\
\hline & $R^{2}$ & 0.88 & 0.85 & 0.12 & 0.91 & 0.34 & 0.65 & 0.78 & 0.93 & 0.93 & 0.97 & 0.97 & 0.94 & 0.77 \\
\hline & Corr & 0.94 & 0.92 & 0.63 & 0.95 & 0.7 & 0.85 & 0.83 & 0.94 & 0.97 & 0.98 & 0.97 & 0.95 & 0.89 \\
\hline & \multicolumn{14}{|c|}{ Lasso regression } \\
\hline & Diff & 0.02 & 0.04 & 0.04 & 0.03 & 0.03 & 0.04 & 0.04 & 0.04 & 0.05 & 0.02 & 0.02 & 0.02 & 0.03 \\
\hline & Std & 0.04 & 0.07 & 0.07 & 0.04 & 0.05 & 0.06 & 0.07 & 0.08 & 0.09 & 0.04 & 0.03 & 0.04 & 0.06 \\
\hline & $R^{2}$ & 0.88 & 0.85 & 0.17 & 0.91 & 0.35 & 0.67 & 0.79 & 0.93 & 0.92 & 0.97 & 0.98 & 0.94 & 0.78 \\
\hline & Corr & 0.94 & 0.92 & 0.64 & 0.95 & 0.7 & 0.86 & 0.83 & 0.94 & 0.97 & 0.98 & 0.98 & 0.95 & 0.89 \\
\hline & \multicolumn{14}{|c|}{ SVR } \\
\hline & Diff & 0.02 & 0.05 & 0.04 & 0.03 & 0.03 & 0.04 & 0.04 & 0.05 & 0.05 & 0.02 & 0.02 & 0.02 & 0.03 \\
\hline & Std & 0.04 & 0.07 & 0.07 & 0.05 & 0.05 & 0.07 & 0.07 & 0.08 & 0.1 & 0.04 & 0.04 & 0.04 & 0.06 \\
\hline & $R^{2}$ & 0.87 & 0.83 & 0.12 & 0.9 & 0.25 & 0.65 & 0.78 & 0.93 & 0.91 & 0.97 & 0.98 & 0.94 & 0.76 \\
\hline & Corr & 0.94 & 0.92 & 0.63 & 0.95 & 0.69 & 0.85 & 0.82 & 0.94 & 0.97 & 0.98 & 0.97 & 0.95 & 0.88 \\
\hline \multirow{15}{*}{ 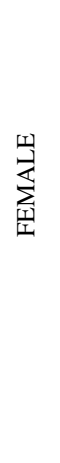 } & \multicolumn{14}{|c|}{ Linear regression } \\
\hline & Diff & 0.02 & 0.03 & 0.03 & 0.02 & 0.03 & 0.03 & 0.02 & 0.03 & 0.03 & 0.03 & 0.02 & 0.02 & 0.03 \\
\hline & Std & 0.04 & 0.05 & 0.06 & 0.03 & 0.05 & 0.04 & 0.03 & 0.05 & 0.05 & 0.07 & 0.03 & 0.03 & 0.04 \\
\hline & $R^{2}$ & 0.82 & 0.86 & 0.10 & 0.89 & 0.09 & 0.78 & 0.91 & 0.91 & 0.91 & 0.53 & 0.96 & 0.96 & 0.73 \\
\hline & Corr & 0.9 & 0.94 & 0.61 & 0.95 & 0.67 & 0.88 & 0.93 & 0.92 & 0.93 & 0.9 & 0.97 & 0.96 & 0.88 \\
\hline & \multicolumn{14}{|c|}{ Lasso regression } \\
\hline & Diff & 0.02 & 0.03 & 0.03 & 0.02 & 0.02 & 0.03 & 0.02 & 0.03 & 0.03 & 0.03 & 0.02 & 0.02 & 0.03 \\
\hline & Std & 0.04 & 0.04 & 0.06 & 0.03 & 0.04 & 0.04 & 0.03 & 0.05 & 0.05 & 0.06 & 0.03 & 0.02 & 0.04 \\
\hline & $R^{2}$ & 0.81 & 0.90 & 0.12 & 0.91 & 0.27 & 0.80 & 0.93 & 0.91 & 0.90 & 0.82 & 0.97 & 0.97 & 0.78 \\
\hline & Corr & 0.91 & 0.94 & 0.61 & 0.96 & 0.67 & 0.88 & 0.93 & 0.91 & 0.93 & 0.92 & 0.97 & 0.96 & 0.88 \\
\hline & \multicolumn{14}{|c|}{ SVR } \\
\hline & Diff & 0.03 & 0.03 & 0.03 & 0.03 & 0.02 & 0.03 & 0.02 & 0.03 & 0.03 & 0.03 & 0.02 & 0.02 & 0.03 \\
\hline & Std & 0.04 & 0.05 & 0.06 & 0.04 & 0.04 & 0.04 & 0.04 & 0.05 & 0.05 & 0.06 & 0.03 & 0.03 & 0.04 \\
\hline & $R^{2}$ & 0.76 & 0.88 & 0.07 & 0.89 & 0.22 & 0.79 & 0.89 & 0.89 & 0.89 & 0.79 & 0.96 & 0.96 & 0.75 \\
\hline & Corr & 0.88 & 0.93 & 0.6 & 0.93 & 0.65 & 0.88 & 0.93 & 0.88 & 0.91 & 0.92 & 0.96 & 0.95 & 0.87 \\
\hline
\end{tabular}

Table 1: Statistical metrics for male and female groups. 


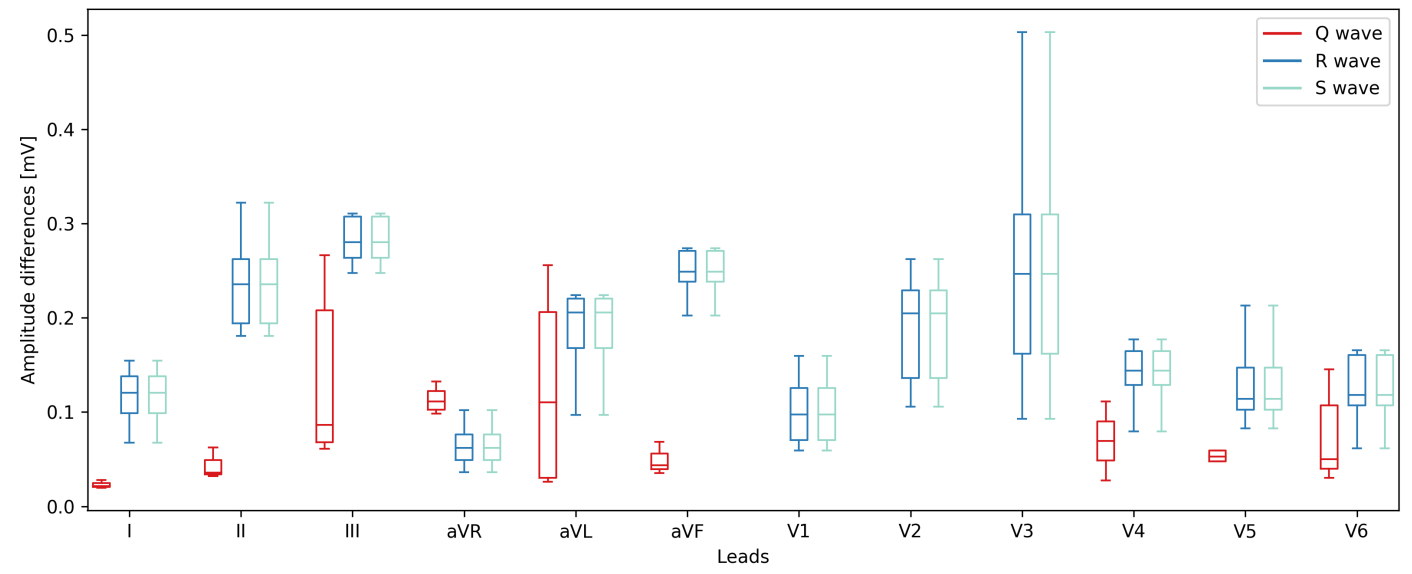

Figure 4: Average amplitude differences between reference and reconstructed signals characteristic points.

Further development will be required in patients with various cardiac abnormalities. It would be critically important to verify the results in patient with morphological abnormalities, including hypertrophies, blocks, ischemia or myocardial infarctions as well as rhythms abnormalities, i.e. atrial fibrillation.

The proposed limb lead placement can possibly provide even fewer artifacts than ML positions, which without signal reconstruction is of poor diagnostic quality [3]. In the future, recording of limb leads closer together on the subject's torso may provide increased usability, while also the conversion matrix can provide a more congruent signal to standard limb lead placement.

\section{Acknowledgments}

The study was supported by SmartMedics Sp. z o. o.

\section{References}

[1] Wilson FN, Kossmann CE, Burch GE, Goldberger E, Graybiel A, Hecht HH, Johnston FD, Lepeschkin E, Myers GB. Recommendations for standardization of electrocardiographic and vectorcardiographic leads. Circulation 1954;10(4):564-573.

[2] Mason RE, Likar I. A new system of multiple-lead exercise electrocardiography. American Heart Journal 1966; 71(2): 196 - 205. ISSN 0002-8703.

[3] Jowett NI, Turner AM, Cole A, Jones PA. Modified electrode placement must be recorded when performing 12-lead electrocardiograms. Postgraduate Medical Journal 2005; 81(952):122 - 125.

[4] Bartosik J, Pahlm O, Edenbrandt L, Svensson J, Haisty WK, Wagner GS. Reconstruction of the standard 12lead ECG from recordings using nonstandard activitycompatible proximal limb lead positions. Journal of Electrocardiology 1995;28(1):33-38. ISSN 00220736.
[5] Man S, Maan AC, Kim E, Draisma HH, Schalij MJ, Van Der Wall EE, Swenne CA. Reconstruction of standard 12lead ECGs from 12-lead ECGs recorded with the MasonLikar electrode configuration. Computers in Cardiology 2007;34:701-704. ISSN 02766574.

[6] Kaewfoongrungsi P, Hormdee D. Linear regression based synthesis of 12-lead ecg system form 5-electrodes using lead v1. Proceedings of The IRES 11th International Conference 2015;

[7] Kaewfoongrungsi P, Hormdee D. Deriving the 12-lead ecg from easi electrodes via nonlinear regression. Proceedings of The IRES 11th International Conference 2015;

[8] Efron B, Trevor Hasti and IJ, Tibshirani R. Least angle regression. The Annals of Statistics 2004;32(2):407 - 499.

[9] Wojciech O, Ewaryst T. Investigation of a transfer function between standard 12-lead ecg and easi ecg. Analysis of Biomedical signals and Images 2010;20:322 - 327. ISSN $1211412 X$.

[10] Yodjaiphet A, Theera-Umpon N, Auephanwiriyakul S. Electrocardiogram reconstruction using support vector regression. 2012 IEEE International Symposium on Signal Processing and Information Technology 2012;ISSN 21627843.

[11] Farrell RM, Syed A, Syed A, Gutterman DD. Effects of limb electrode placement on the 12- and 16-lead electrocardiogram. Journal of Electrocardiology 2008;41(6):536 545. ISSN 0022-0736.

Address for correspondence:

Judyta Salamon

Faculty of Physics

Warsaw University of Technology

Koszykowa 75, 00-622, Warsaw, Poland

judyta.salamon.dokt@pw.edu.pl 